Supplementary Information (Mathematica Notebook) for:

\title{
Polarizable Atomic Multipole X-Ray Refinement: Application to Peptide Crystals
}

\author{
Michael J. Schnieders, Timothy D. Fenn, Vijay S. Pande \\ and Axel T. Brunger
}

Department of Chemistry, Stanford CA, 94305, USA; Department of Molecular and Cellular Physiology, Stanford CA, 94305, USA; Howard Hughes Medical Institute, USA.

\section{E-mail: schnied@stanford.edu}

Define a term of the spherical and anisotropic scattering density given in Eq. (6).

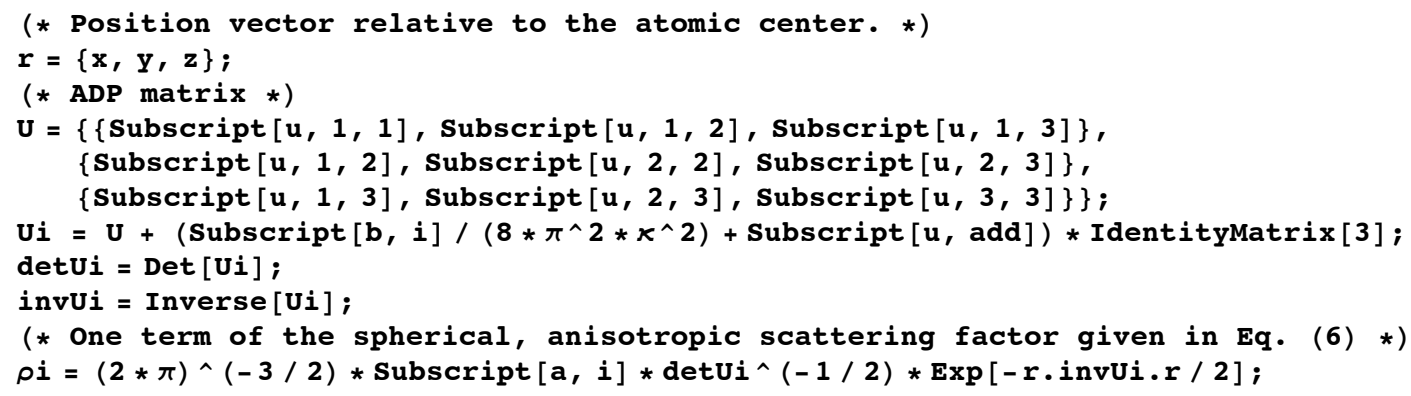




\section{Construct and plot example aspherical and anisotropic scattering densities as given in Eq. (16).}

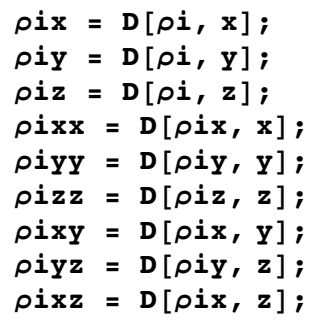


Verify analytic forms of the Cartesian Gaussian multipole tensors from Eq. (18).

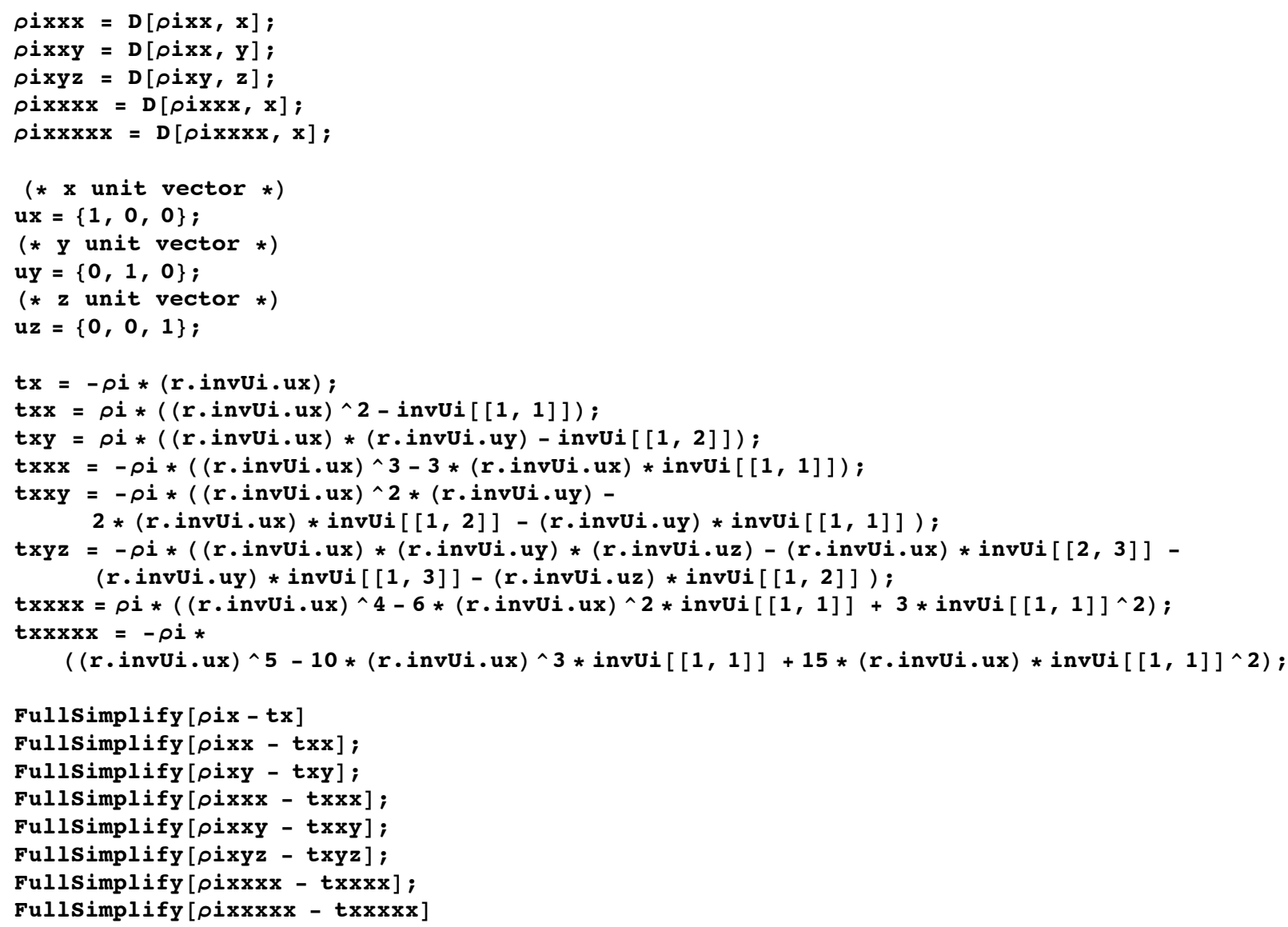




\section{Verify analytic derivatives of the Cartesian Gaussian multipole tensors with respect to ADPs given in Eq. (28).}

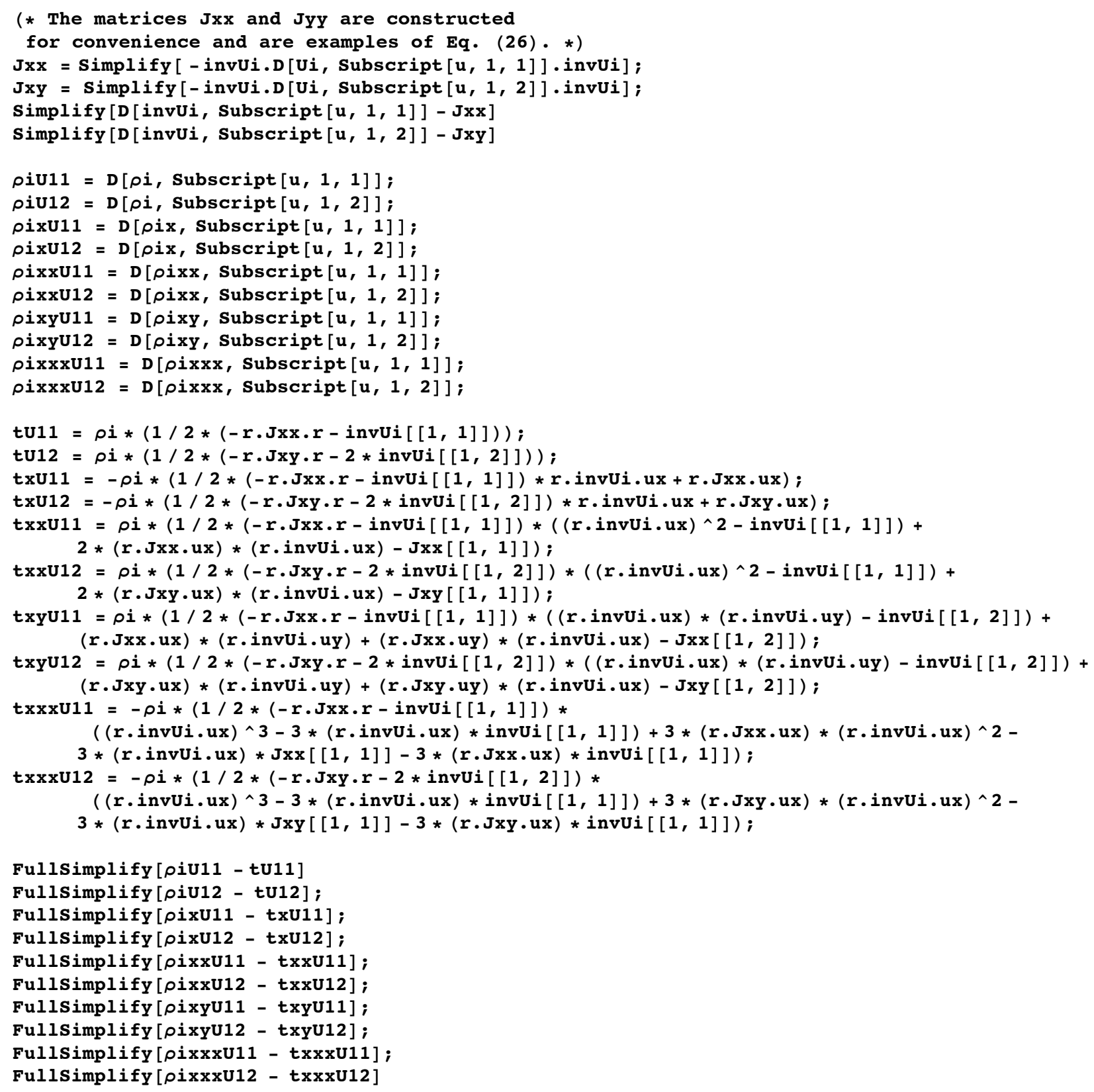




\section{Verify analytic derivatives of the Cartesian Gaussian multipole tensors with respect to $\kappa$ given in Eq. (34).}

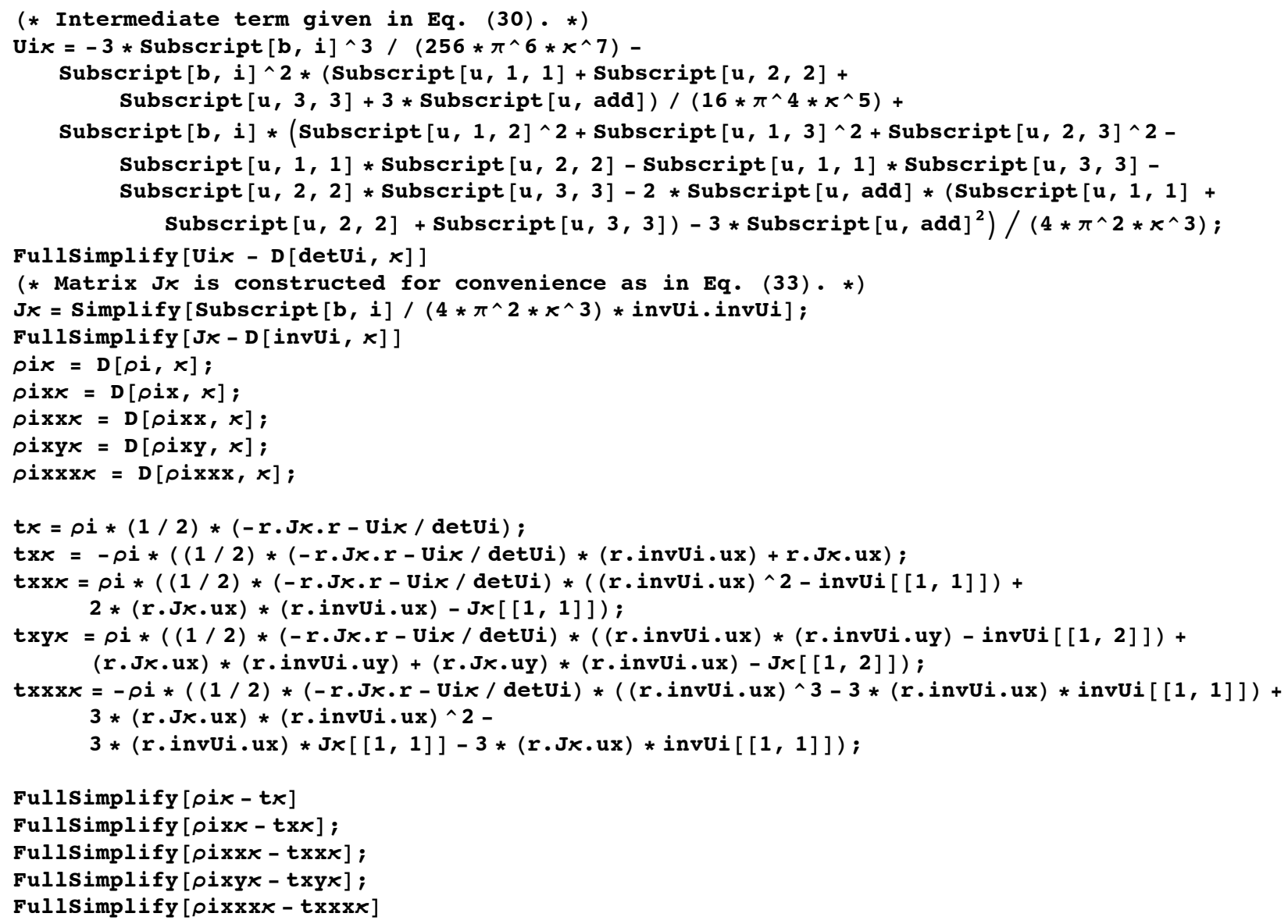

